Gut, 1988, 29, 752-755

\title{
Effect and mechanism of action of isosorbide-5-mononitrate
}

\author{
P C HAYES, D WESTABY, AND ROGER WILliaMS \\ From the Liver Unit, King's College Hospital and School of Medicine and Dentistry, London
}

SUMMARY Nitrates have been shown to decrease portal pressure in cirrhotic patients with portal hypertension and this has been attributed to decreased portal venous resistance. We studied the effect and mechanism of action of oral administration of isosorbide-5-mononitrate (Is-5-Mn) $(20 \mathrm{mg})$, which, unlike the dinitrate, does not require hepatic biotransformation to a vasoactive metabolite on portal and systemic haemodynamics in 11 patients with portal hypertension complicating cirrhosis. A significant reduction in portal pressure gradient (WHVP-FHVP) (from $23.9(3.4)$ to $21.8(3.4) \mathrm{mmHg}: \mathrm{p}<0.005)$ occurred 60 minutes after Is-5-Mn due entirely to a fall in WHVP, associated with decreased estimated liver blood flow (from 1940 (159) to 1639 (179) $\mathrm{ml} / \mathrm{min}$ : $\mathbf{p}<0 \cdot 05$ ). Right atrial and pulmonary artery pressures and cardiac index fell significantly whilst mean arterial pressure remained unaffected. Heart rate and the calculated systemic vascular resistance index increased significantly. Significant correlations existed between the reduction in portal pressure gradient and fall in cardiac index $(r=0.65, p<0.05)$ and increase in systemic vascular resistance index $(r=0.72, p<0.02)$. The observed decrease in estimated liver blood flow, in association with an increase in systemic vascular resistance index, suggests that baroreceptor mediated splanchnic vasoconstriction may be one of the factors responsible for the fall in portal pressure, rather than portal venous dilatation.

Portal pressure can be reduced by three mechanisms: a reduction in portal venous inflow, an increase in portal venous compliance and a reduction in portal outflow resistance. Pharmacological reduction in portal pressure has been achieved using such agents as propranolol, ${ }^{1}$ prazocin, ${ }^{2}$ verapamil, ${ }^{3}$ atenolol, ${ }^{2}$ metoprolol, ${ }^{+}$and the B2 antagonist ICI 118551. ${ }^{5}$ All these agents are believed to act by reducing portal inflow either by reducing cardiac output or by causing splanchnic vasoconstriction.

Recent evidence that nitrates also decrease portal pressure in patients with cirrhosis ${ }^{\alpha-7}$ is supported by animal studies which suggested that its mechanism of action may be to directly reduce portal vascular resistance. ${ }^{8}$ The present study was undertaken to investigate the effect and in particular the mechanism of action of nitrates on portal and systemic haemodynamics in man. Isosorbide-5-mononitrate (Is-5$\mathrm{Mn}$ ) has not previously been investigated but offers

Address for correspondence: Dr Roger Williams, Liver Unit, King's College Hospital, Denmark Hill, London SE5 9RS.

Received for publication 12 January 1988. theoretical advantages over the dinitrates in patients with liver disease as it does not require hepatic biotransformation to a vasoactive metabolite, ${ }^{9}$ does not accumulate in liver disease and exhibits complete bioavailability."

\section{Methods}

PATIENTS

Eleven patients (five men, six women) with portal hypertension and biopsy proven cirrhosis (eight alcoholic cirrhosis and three primary biliary cirrhosis) were studied with informed consent. The study was approved by the ethical committee at King's College Hospital and Medical School. Their liver function, categorised according to the Pugh modification of Child's classification, was grade $\mathrm{C}$ in 5 of the 11 patients and grade $B$ in the remainder. Nine of the patients were studied after a minimum of 72 hours after control of a variceal haemorrhage whilst the other two were undergoing haemodynamic investigations before paracentesis for ascites. 

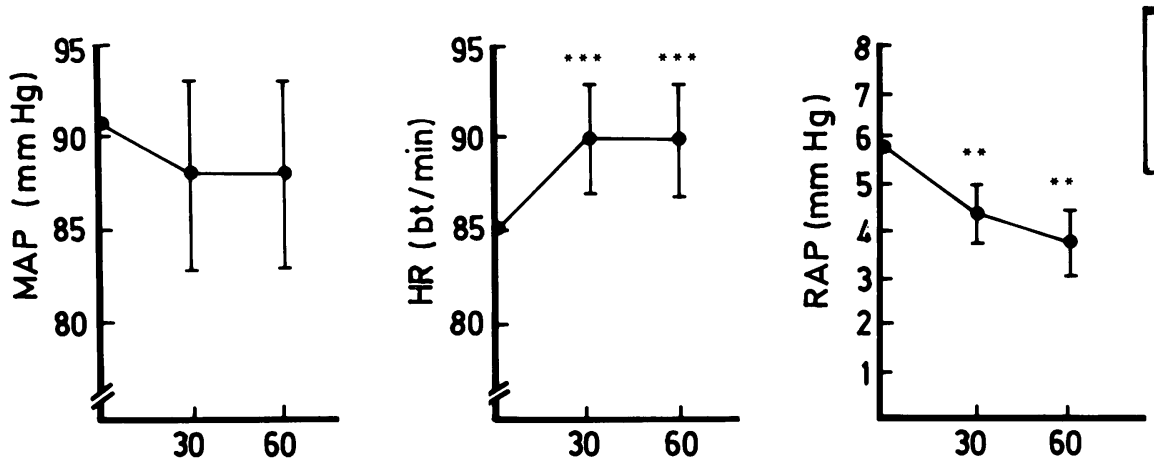

$$
\begin{aligned}
& * p<0.005 \\
& \cdots p<0.01 \\
& * p<0.05
\end{aligned}
$$
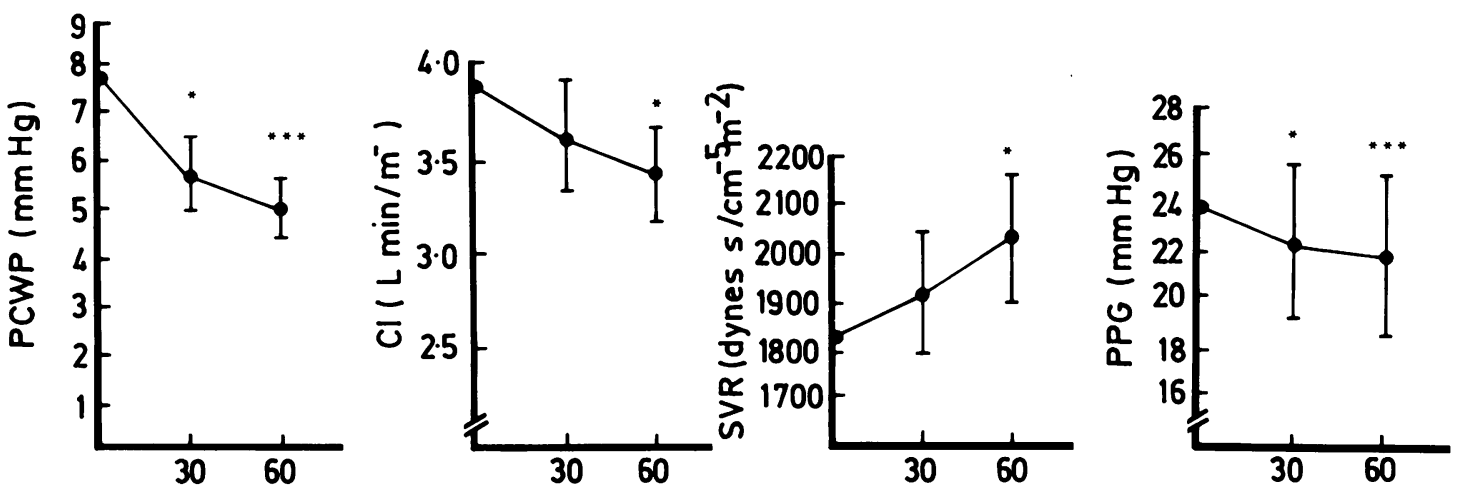

Fig. 1 Haemodynamic changes occurring with Is-5-Mn after 30 and 60 minutes.

The patients were examined after an overnight fast. A Swann-Ganz pulmonary flotation catheter and an hepatic venous balloon catheter were inserted through the right subclavian and femoral veins respectively. Estimated liver blood flow (ELBF) was measured by the indocyanine green constant infusion method" before and 60 minutes after the oral administration of $20 \mathrm{mg}$ of Is-5-Mn.

The baseline haemodynamic parameters measured were: heart rate (bt/min) (electrocardiographically); right atrial, pulmonary artery and pulmonary capillary wedge pressures $(\mathrm{mmHg})$ (RAP, PAP, and PCWP respectively); wedged and free hepatic venous pressures (mmHg) (WHVP and FHVP); cardiac output $(1 / \mathrm{min})$ (by thermodilutation) and mean arterial blood pressure (measured sphygmomanometrically and calculated as the diastolic blood pressure $+1 / 3$ the pulse pressure). The midaxillary line was used as the external zero reference point. The measurements were repeated 30 and 60 minutes after the administration of the drug.

Systemic vascular resistance index (SVRI) was calculated using the formula:

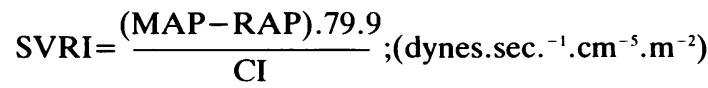

The portal pressure gradient (PPG) was calculated from the WHVP minus the FHVP and the hepatic vascular resistance (HVR) using the formula:

$$
\mathrm{HVR}=\frac{\mathrm{PPG}}{\mathrm{ELBF}} ;\left(\text { dynes } \cdot \mathrm{sec}^{-1} \cdot \mathrm{cm} \cdot^{-5} \cdot\right)
$$

STATISTICAL ANALYSIS

Analysis of the differences between values before and after Is-5-Mn was made using a two tailed paired Student's $t$-test and correlations between changes were made by regression analysis. All values are expressed as mean (SE).

\section{Results (Figure 1 and Table)}

Changes in splanchnic haemodynamics were seen by 30 minutes with significant reductions in WHVP, FHVP, and PPG. The fall in FHVP, however, was not maintained at 60 minutes, unlike the alterations in WHVP and PPG. Measurements of liver blood flow were unable to be obtained in five patients because of poor hepatic extraction and/or failure to reach equilibrium. In the remaining six patients, however, a significant reduction in estimated liver blood flow was observed when measured 60 minutes 
Table Haemodynamic changes before and 30 and 60 minutes after isosorbide-5-mononitrate

\begin{tabular}{|c|c|c|c|c|c|}
\hline & 0 & $p^{*}$ & 3 & $p^{\dagger}$ & 60) \\
\hline HR & $85 \cdot 0(3 \cdot 0)$ & $<0.005$ & $89 \cdot 8(3 \cdot())$ & $<0) \cdot(0) 5$ & $89 \cdot 7(3 \cdot(1)$ \\
\hline MAP & $9(0 \cdot 0(4 \cdot 0)$ & NS & $88 \cdot 0(5 \cdot())$ & NS & $88.9(5.0)$ \\
\hline RAP & $5 \cdot 7(1 \cdot 0)$ & $<0 \cdot(01$ & $4 \cdot 3(0 \cdot 6)$ & $<() \cdot() 1$ & $3 \cdot 7(0 \cdot 7)$ \\
\hline PAP & $11.7(1.1)$ & $<0 \cdot 01$ & $9 \cdot 6(0 \cdot 8)$ & $<0.001$ & $8 \cdot 8(0.9)$ \\
\hline PCWP & $7 \cdot 6(1 \cdot 0)$ & $<0 \cdot(05$ & $5 \cdot 7(0 \cdot 7)$ & $<0 .(005$ & $5 \cdot 0(0 \cdot 6)$ \\
\hline $\mathrm{Cl}$ & $3.89(0.33)$ & NS & $3.62(0 \cdot 27)$ & $<0) \cdot(15$ & $3.42(0.24)$ \\
\hline SVRI & $1831(141)$ & NS & $1911(133)$ & $<0 .(1) 5$ & $2030(136)$ \\
\hline WHVP & $33 \cdot 3(2 \cdot(19)$ & $<0 \cdot(0) 5$ & $30 \cdot 2(3 \cdot(0)$ & $<0 \cdot(0) 5$ & $29 \cdot 8(3 \cdot 1)$ \\
\hline FWVP & $9.4(1 \cdot 1)$ & $<0 \cdot(01$ & $7 \cdot 8(1 \cdot 3)$ & NS & $8.0(1.4)$ \\
\hline PPG & $23.9(3.4)$ & $<0.05$ & $22 \cdot 4(3 \cdot 2)$ & $<0.05$ & $21 \cdot 8(3.4)$ \\
\hline ELBF & $1940(159)$ & & & $<0 \cdot(0) 5$ & $1639(179)$ \\
\hline HVR & $11 \cdot 4(2 \cdot 6)$ & & & NS & $12 \cdot(0(2 \cdot 9)$ \\
\hline
\end{tabular}

All values are mean (SE); HR, heart rate (beats/min); MAP, mean arterial pressure: RAP, right atrial pressure; PAP. pulmonary artery pressure; PCWP, pulmonary capillary wedge pressure; $\mathrm{CI}$, cardiac index $\left(1 . \mathrm{min}^{-1} \cdot \mathrm{m}^{2}\right)$; SVRI, systemic vascular resistance index (dynes.sec '. $\mathrm{cm}^{5} \cdot \mathrm{m}^{-2}$ ): WHVP, wedged hepatic venous pressure; FHVP, free hepatic venous pressure; PPG, portal pressure gradient ELFB, estimated liver blood flow; HVR, hepatic vascular resistance (dynes.sec. ${ }^{1} . \mathrm{cm}^{-5}$. All pressure measurements in $\mathrm{mmHg}$. ${ }^{*}(0-30$ values; $+0-60$ values

after administration of the drug whilst no change was seen in hepatic vascular resistance. A reduction in portal pressure gradient was seen in 8 of the 11 patients with a mean overall fall of $2.09(0.56)$ $\mathrm{mmHg}$.

Changes in systemic haemodynamics were seen by 30 minutes with significant reductions in RAP, PAP, PCWP associated with a significant increase in heart rate. The decrease in cardiac index was only shown 60 minutes after administration of the drug as was the increase in systemic vascular resistance index. No change in mean arterial blood pressure was shown at any time during the study.

The fall in portal pressure correlated with the rise in SVRI $(r=0.72 ; p<0 \cdot 02)$ and weakly with the fall in $\mathrm{CI}(\mathrm{r}=0.65 ; \mathrm{p}<0.05)$ but not between changes in portal pressure gradient and PCWP or RAP. Similarly no correlation was seen between PPG and ELBF.

\section{Discussion}

This study confirms that the oral administration of Is-5-Mn $(20 \mathrm{mg})$, the standard single dose used in cardiological practice, is effective $(p<0 \cdot 005)$ in reducing portal pressure. The fall in portal pressure gradient was accompanied by, and correlated with, a reduction in the cardiac index and rise in SVRI. The predominant systemic haemodynamic changes produced by the drug were reductions in cardiac preload - that is PCWP, PAP, and RAP, whilst no change
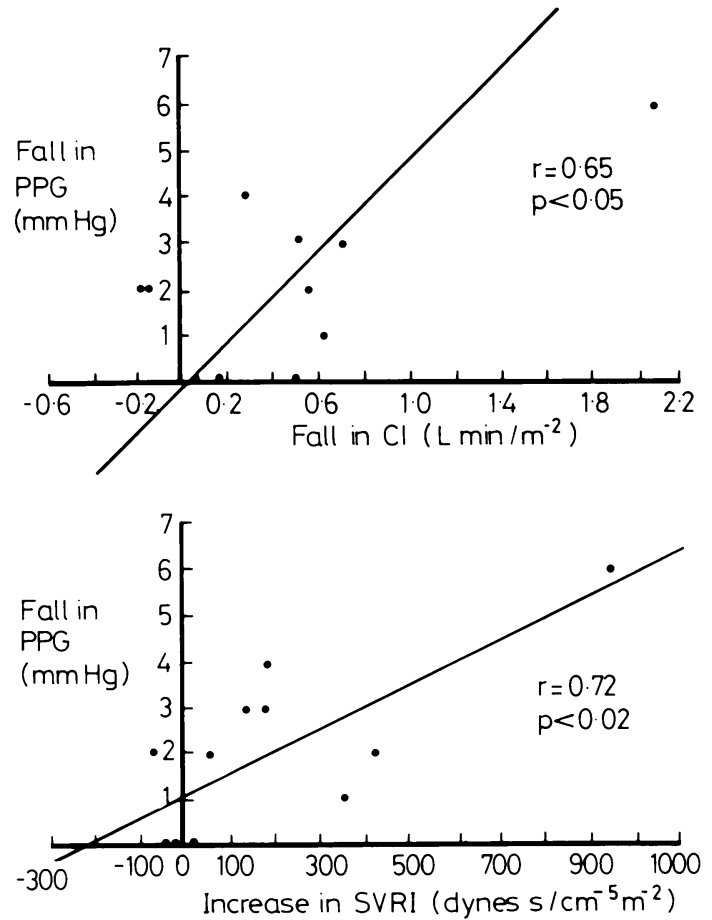

Fig. 2 Correlations between the reduction in portal pressure and fall in cardiac index and increase in systemic vascular resistance index.

was seen in mean arterial pressure. Although the measurements were recorded over a 60 minute period spontaneous changes over this period are known to be minor with no significant change in portal pressure gradient, liver blood flow and cardiac index." Other haemodynamic changes would be expected to be in the opposite direction to those observed here - that is, decrease in heart rate and SVRI. It seems likely that the major action of Is-5-Mn is to produce venodilatation, resulting in a reduction in venous return. The resultant reduction in left ventricular filling pressure would activate a baroreceptor mediated reflex resulting in the observed rise in systemic vascular resistance with maintenance of blood pressure. The reflex increase in arterial resistance occurring in the splanchnic vessels would produce the observed reduction in liver blood flow and portal pressure:

The present results support the findings of a recent study in animals reported by Blei and Gottstein where an increase in SVRI was seen with low dose nitrate therapy although in the rat they were not able to identify decreases in portal pressure despite a fall in portal venous inflow. A decrease in portal pressure was only detected by those authors with larger doses 
at the expense of a reduction in arterial pressure. An increase in SVRI was not seen using this larger intravenous dosage.

This dose dependent effect of the nitrates may be responsible for the differences in published studies. ${ }^{3613}$ Although small and large doses appear capable of reducing portal pressure the mechanism whereby it takes place differs, the smaller dose affecting primarily cardiac preload, with a secondary rise in SVRI, whilst larger doses directly affect the arterial circulation, reducing portal pressure by lowering systemic and hence splanchnic perfusion pressure.

The high percentage of responders with respect to a reduction in portal pressure suggests that Is-5-Mn may deserve further assessment as a possible therapeutic agent alone or in combination with wother 'active agents', although it is important to stress that there is continued controversy as to whether a fall in portal pressure results in a decreased risk of variceal bleeding. Furthermore, any proposed long term use of nitrates for the management of portal hypertension should be tempered by the well documented development of tolerance found in cardiological practice. ${ }^{4}$

\section{References}

1 Lebrec D, Nouel O, Corbic M, Benhamou J-P. Propranolol - a medical treatment for portal hypertension? Lancet 1980; ii: 180-2.

2 Mills PR, Rae AP, Farah DA, et al. Comparison of three adrenoreceptor blocking agents in patients with cirrhosis and portal hypertension. Gut 1984; 25 : 73-8.

3 Freeman JG, Barton JR, Record CO. Effect of isosorbide dinitrate, verapamil and labetalol on portal pressure in cirrhosis. Br Med J 1985; 291: 561-2.
4 Westaby D, Bihari D, Gimson AES, et al. Selective and non-selective beta blockade in the reduction of portal hypertension in patients with cirrhosis and portal hypertension. Gut 1984; 25: 121-4.

5 Bihari D, Westaby D, Gimson AES, et al. Reductions in portal pressure by selective $\mathrm{B}_{2}$-adrenoreceptor blockade in patients with cirrhosis and portal hypertension. $\mathrm{Br} J$ Clin Pharmacol 1983; 17: 753-7.

6 Groszmann RJ, Kravetz D, Bosch J, et al. Nitroglycerin improves the hemodynamic response of vasopressin in portal hypertension. Hepatology 1982; 2: 757-62.

7 Westaby D, Gimson AES, Hayes PC, et al. Haemodynamic response to intravenous vasopressin and nitroglycerin in portal hypertension. Gut 1988; 29: 372-7.

8 Blei AT, Gottstein J. Isosorbide dinitrate in experimental portal hypertension: a study of factors that modulate the hemodynamic response. Hepatology 1986 ; 6: 107-11.

9 Chasseaud LF. Pharmacokinetics and bioavailability of different nitrate preparations. $\mathrm{Br} J$ Clin Pract (Symposium suppl) 1983; 26: 7-14.

10 Abshagen U. Overview of pharmacokinetics of IS-5-MN. In: Abstracts of the Second International Symposium on Mononitrates. Berling: Boehringer Mannheim, 1986.

11 Bosch J, Kravetz D, Rodes J. Effects of somatostatin on hepatic and systemic hemodynamics in patients with cirrhosis of the liver: comparison with vasopressin. Gastroenterology 1981; 80: 518-27.

12 Lebrec D, Hillon P, Munoz C, et al. The effect of propranolol on portal hypertension in patients with cirrhosis: a hemodynamic study. Hepatology 1982; 2: 523-7.

13 Dawson J, Gertsch P, Mosimann F, et al. Endoscopic variceal pressure measurements: response to isosorbide dinitrate. Gut 1985; 26: 843-7.

14 Silber S. Clinical relevance of nitrate tolerance. In: Cophn JN, Rittinghausen R, eds. Mononitrates. Berlin: Springer-Verlag, 1984: 130-46. 